\title{
Development of a neutral embedding resin for optical imaging of fluorescently labeled biological tissue
}

Hongfu Zhou

Yadong Gang

Shenghua Chen

Yu Wang

Yumiao Xiong

Longhui Li

Fangfang Yin

Yue Liu

Xiuli Liu

Shaoqun Zeng 


\title{
Development of a neutral embedding resin for optical imaging of fluorescently labeled biological tissue
}

\author{
Hongfu Zhou, a,b,t Yadong Gang, ${ }^{a, b, \dagger}$ Shenghua Chen, ${ }^{a, b}$ Yu Wang, ${ }^{a, b}$ Yumiao Xiong, ${ }^{a, b}$ Longhui Li, ${ }^{a, b}$ \\ Fangfang Yin, ${ }^{a, b}$ Yue Liu, ${ }^{a, b}$ Xiuli Liu, ${ }^{a, b}$ and Shaoqun Zeng, ${ }^{a, b, *}$ \\ ${ }^{a}$ Huazhong University of Science and Technology, Collaborative Innovation Center for Biomedical Engineering, Wuhan National Laboratory for \\ Optoelectronics, Wuhan, Hubei, China \\ ${ }^{b}$ Huazhong University of Science and Technology, School of Engineering Sciences, Britton Chance Center and MOE Key Laboratory for \\ Biomedical Photonics, Wuhan, Hubei, China
}

\begin{abstract}
Plastic embedding is widely applied in light microscopy analyses. Previous studies have shown that embedding agents and related techniques can greatly affect the quality of biological tissue embedding and fluorescent imaging. Specifically, it is difficult to preserve endogenous fluorescence using currently available acidic commercial embedding resins and related embedding techniques directly. Here, we developed a neutral embedding resin that improved the green fluorescent protein (GFP), yellow fluorescent protein (YFP), and DsRed fluorescent intensity without adjusting the $\mathrm{pH}$ value of monomers or reactivating fluorescence in lye. The embedding resin had a high degree of polymerization, and its fluorescence preservation ratios for GFP, YFP, and DsRed were $126.5 \%$, 155.8\%, and 218.4\%, respectively. @ 2017 Society of Photo-Optical Instrumentation Engineers (SPIE) [DOI: 10.1117/1.JBO.22.10.106015]
\end{abstract}

Keywords: biomaterials; polymers; biological tissue; confocal microscopy; two-photon microscopy; fluorescence.

Paper 170437LR received Jul. 5, 2017; accepted for publication Oct. 2, 2017; published online Oct. 27, 2017.

Plastic embedding of large samples is important for high-resolution three-dimensional (3-D) imaging, and the embedding reagent used plays a key role in the microimaging of biological tissue. $^{1-3}$ To improve the axial resolution in imaging large embedded samples, fluorescence microscopy and electron microscopy are often combined with serial sectioning. ${ }^{4-7}$ Therefore, the embedding resin must have adequate hardness to enable the slicing of thin sections. There are two categories of plastic embedding agents available at present. The first category of embedding resin is epoxy resin, which is cured with acid anhydride under a tertiary amine catalyst. Epoxy is usually used for sample embedding for electron microscopy owing to its high autofluorescence and high polymerization temperature, which quenches fluorescence irreversibly. Thus, it is not suitable for embedding fluorescent samples. ${ }^{8-10}$ The second category of embedding resin is acrylic resin, which is polymerized under an azo-based or peroxide-based initiator and is successfully used in the plastic embedding field because of its low auto-fluorescence and simple embedding technique. ${ }^{11-14}$ Due to the high acidity of some acrylic monomers, substantial loss of fluorescence in endogenous fluorescent samples occurs after acrylic resin embedding. ${ }^{15,16}$ The fluorescence in endogenous fluorescent samples can be preserved perfectly after embedding in hydroxypropyl methacrylate resin, although this embedding technique requires more than two weeks to permeate a whole mouse brain, which greatly limits its application in large sample embedding. ${ }^{17}$ In all successful commercialized resins, the fluorescence preservation ratio of GMA ( $\mathrm{pH}$ 6.0) was the best, and its fluorescence preservation ratio was $70 \%$; in contrast, the

*Address all correspondence to: Shaoqun Zeng, E-mail: sqzeng@mail.hust. edu.cn

†These authors contributed equally to this work. fluorescence preservation ratio of Unicryl resin was $51.5 \%$, and the fluorescence preservation ratio of LR white resin was $27.6 \% .^{18}$ To overcome the fluorescence loss in endogenous fluorescent proteins after acrylic resin embedding, adding organic bases to the acrylic monomers before resin embedding can be beneficial in preserving endogenous fluorescence, although this reduces the degree of polymerization and causes the resin block to become brittle and difficult to section. ${ }^{18,19}$ The fluorescence of resin-embedded samples is reactivated in lye, which is also used to preserve endogenous fluorescence, although longterm soaking in lye results in swelling of the resin. ${ }^{20}$ Therefore, it is difficult to maintain endogenous fluorescence and adequate hardness simultaneously using currently available acrylic embedding resin and related embedding techniques.

Reactive embedding hydrogels have been widely used in neuroscience research because they maintain the original morphology of the biological tissue and preserve endogenous fluorescence. ${ }^{21-23}$ Therefore, in this study, we aimed to improve the preservation of endogenous fluorescence and the compatibility between the resin and biological tissue by developing an acrylic embedding resin that formed a crosslinked network structure and had a $\mathrm{pH}$ value close to neutral. The epoxy groups on the polymer side chains crosslinked with the residual amino groups of biological tissue, ${ }^{24,25}$ and the degree of polymerization was increased to achieve the appropriate hardness.

The main components of the neutral acrylic embedding resin included glycidyl methacrylate, ethyl methacrylate, and ethylene glycol dimethacrylate. Monomers of glycidyl methacrylate and ethyl methacrylate were mixed with the crosslinking agent ethylene glycol dimethacrylate at a ratio of $6: 3: 1$. Then, $0.2 \%$ 2,2'-azobis(2,4-dimethyl) valeronitrile (ABVN) and $0.05 \%$ triphenylphosphine were added to the mixture, which was 

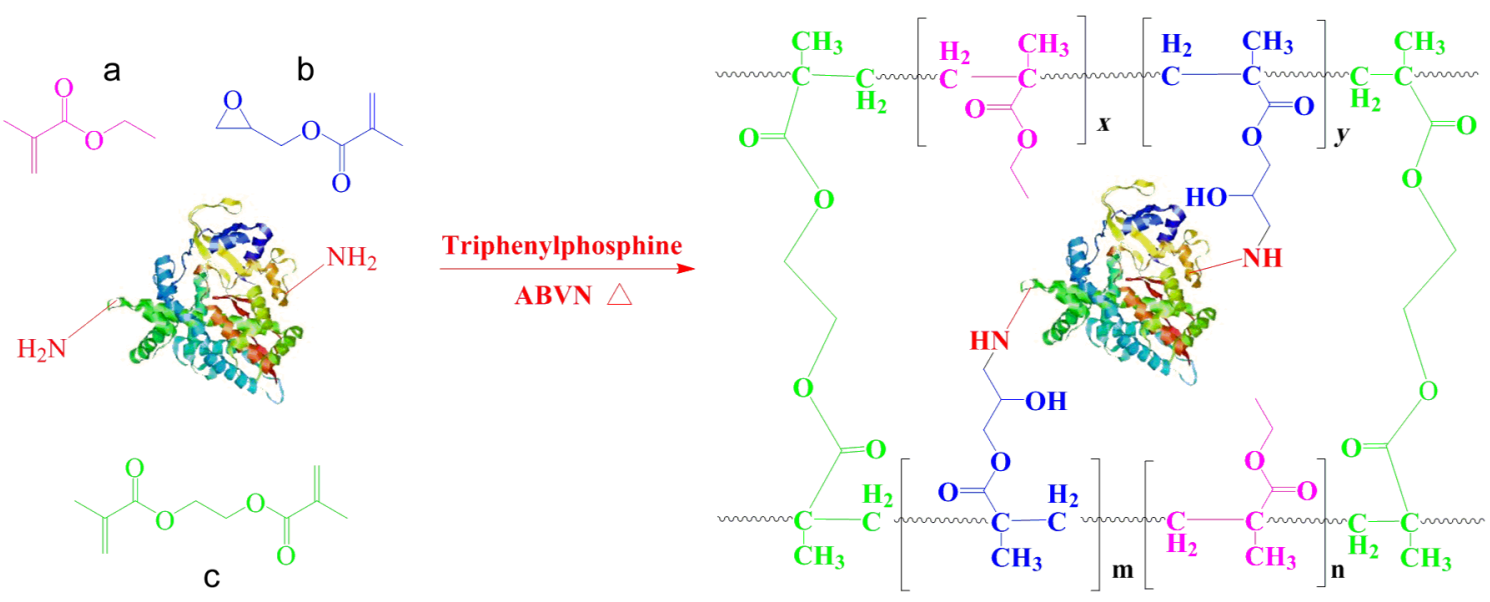

Fig. 1 Polymerization of the neutral embedding resin, and the reaction between the epoxy group of the side chain and the residual amine group of the biological tissue. The structure $a$ is monomer ethyl methacrylate, the structure $b$ is monomer glycidyl methacrylate, the structure $c$ is the crosslinking agent triethylene glycol dimethacrylate, and the protein structure represents the embedded biological tissue.

subsequently stirred magnetically for $20 \mathrm{~min}$ (rotor speed $400 \mathrm{rpm}$ ), the mixture was then stored in the dark at $-10^{\circ} \mathrm{C}$. Glycidyl methacrylate and ethyl methacrylate were copolymerized using the radical initiator $\mathrm{ABVN}$ to form the rigid main chain, and ethylene glycol dimethacrylate was used as the crosslinking agent to form a crosslinked network structure. The epoxy group of glycidyl methacrylate reacted with the residual amine group of the biological tissue in the presence of the catalyst triphenylphosphine to generate hydroxyl groups (Fig. 1).

In order to test the preservation of endogenous fluorescence after embedding with the neutral resin, we used brain slices that were $80 \mu \mathrm{m}$ from the brains of Thy1-GFPM, Thy1-YFPH, and $\mathrm{RV}-\Delta \mathrm{G}-\mathrm{DsR}$ ed mice to quantitatively compare the same cell bodies in their original and resin-embedded states (Fig. 2). Eight-week-old male transgenic mice expressing green fluorescent protein (GFP; Thy1-GFPM line) or yellow fluorescent protein (YFP; Thy1-YFPH) were used in this experiment to compare the fluorescence intensities before and after embedding. Eight-week-old C57BL/6J male mice were used for virus labeling experiments, in which an RV- $\Delta$ G-DsRed virus was used to label neurons. For this, the C57BL/6J mice were first anesthetized using sodium pentobarbital and then fixed on a 3-D fixing device. Next, $300 \mathrm{~nL}$ RV- $\Delta$ G-DsRed virus was injected into the mouse motor cortex (A-P: $1.18 \mathrm{~mm}$; M-L: $1.5 \mathrm{~mm}$; D-V: $1.3 \mathrm{~mm}$ ), determined with reference to the Allen mouse brain map, ${ }^{26}$ and the mice were returned to their home cage for 12 days. Samples from the DsRed-labeled C57BL/6J mice were also used to compare the fluorescence intensity before and after embedding. Mice were perfused according to the recommended protocol for the perfusion of mice. ${ }^{27}$ Animal care and use were in accordance with the guidelines of the Administration Committee of Affairs Concerning Experimental Animals in Hubei Province of China.

The protocol was approved by the Committee on the Ethics of Animal Experiments of the Huazhong University of Science and Technology (Permit Number: 00027340). All efforts were made to minimize the suffering of the animals. The postfixed mouse brains were rinsed three times with $0.01 \mathrm{M}$ phosphate-buffered saline (PBS) for $8 \mathrm{~h}$ each at $4^{\circ} \mathrm{C}$ in the dark. The tissue was then sectioned into $80-\mu \mathrm{m}$ thickness coronal slices using a vibrating microtome (Leica, VT $1000 \mathrm{~S}$ ), and the slices were stored at $4^{\circ} \mathrm{C}$ in the dark. The brain slices were dehydrated according to the following procedure: $75 \%$ tetrahydrofuran (THF) for $3 \mathrm{~min}, 100 \%$ THF for $3 \mathrm{~min}$, and $100 \%$ THF for $4 \mathrm{~min}$. The brain slices were then placed in the resin mixture twice for $5 \mathrm{~min}$ each time. Two drops of resin mixture were then added to the slides, which were subsequently coverslipped. The brain slices on the slides were polymerized in an oven at $40^{\circ} \mathrm{C}$ for $8 \mathrm{~h}$. The results indicated that preservation of the three different types of endogenous fluorescence all increased after embedding with the neutral resin. This experiment was carried out to verify the endogenous fluorescence intensity before and after embedding in the neutral embedding resin $(n=5)$. First, an $80-\mu \mathrm{m}$ thickness brain slice was placed on a slide and coverslipped. Confocal fluorescence microscopy (Zeiss 780) was then used to image the region of interest. The imaged slices were embedded according to the above procedure, and confocal fluorescence microscopy (Zeiss 780) was again used to image the same region using the same parameters. To quantitatively evaluate the endogenous fluorescence intensity of the tissue before and after embedding in the neutral embedding resin, we used the fluorescence intensity of the soma as an indicator. First, the imaging data were imported into Image J software. Within this software, the rectangular-selection tool was activated, a circular area on the soma was selected, and the histogram tool was then used to measure the average gray value; the average gray value of the soma before embedding was denoted as $A$, and the average gray value of the soma after embedding was denoted as $B$. The fluorescence preservation ratio after embedding was calculated as follows: $B / A \times 100 \%$. For each group of experiments, we selected 10 somata to evaluate the average gray values. The fluorescence preservation ratios of YFP, GFP, and DsRed were $126.5 \%, 155.8 \%$, and $218.4 \%$, respectively.

In order to quantitatively evaluate the distortion of the tissue before and after embedding in the neutral embedding resin, we amplified and rotated the embedded image [part of Fig. 2(d)] to match the image before embedding [part of Fig. 2(a)] and then we merged these two images based on three pairs of neurons in the two images. Also, we tracked the morphological structure manually and then merged these two tracked images, then selected nine pairs of same branch of nerve fibers and calculated 

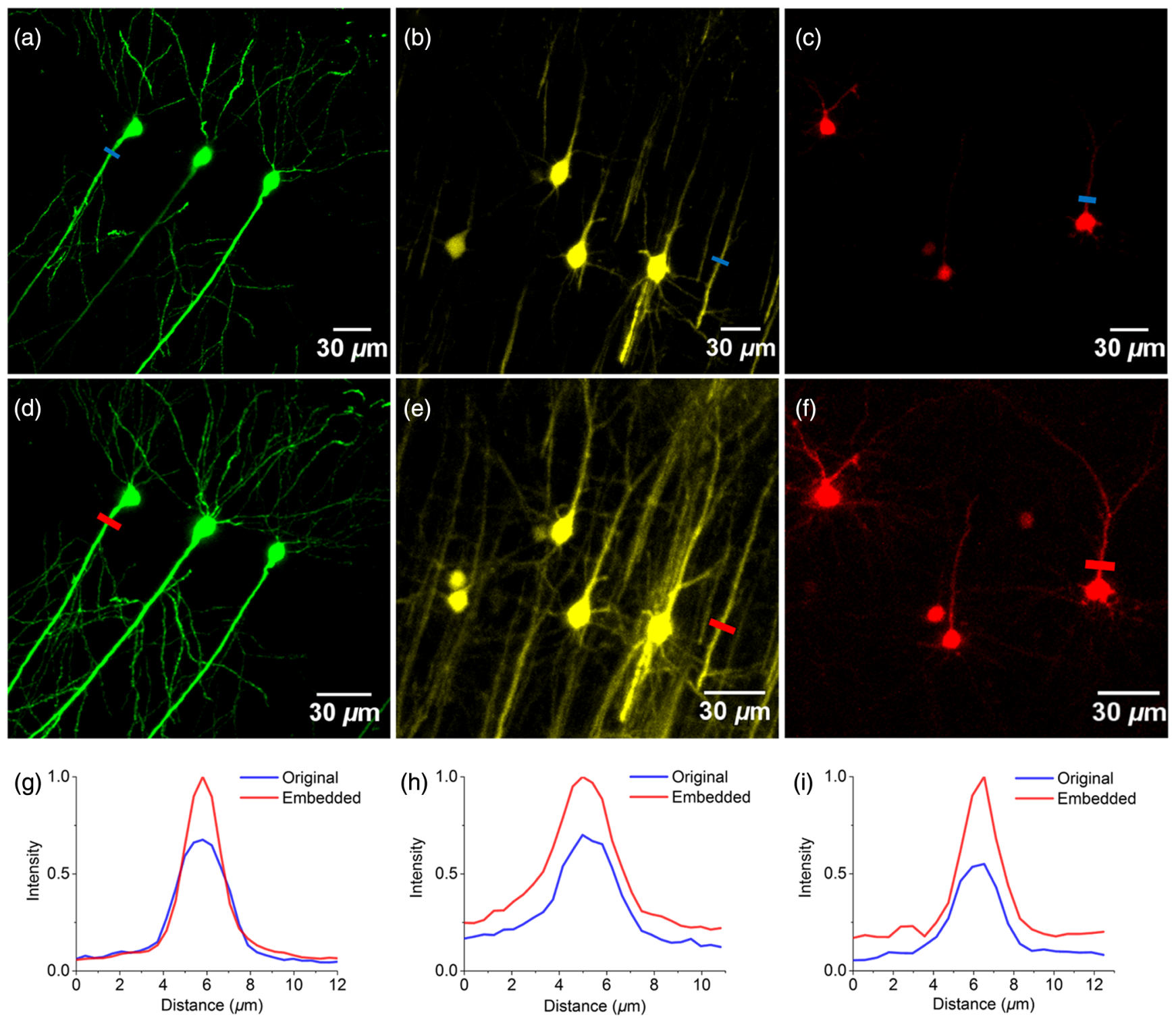

(j) 은 $300 \%$
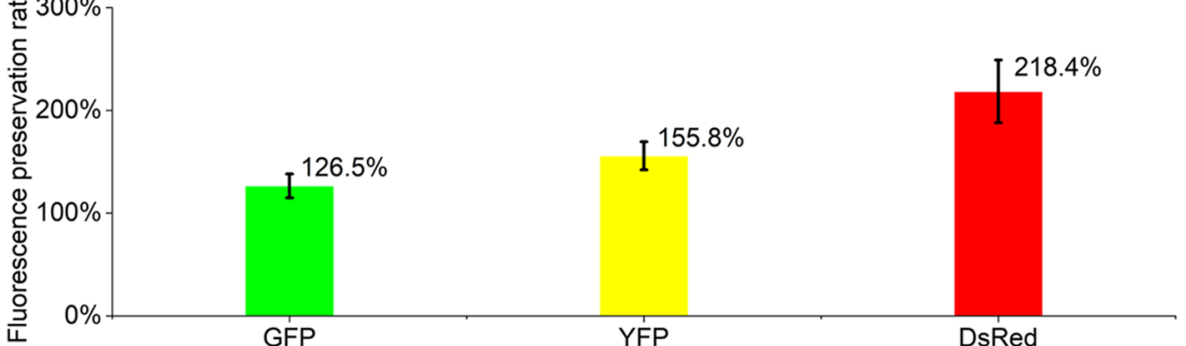

Fig. 2 Preservation of cell structure and GFP and its variants in tissue in the original state and following embedding in neutral resin. (a) GFP fluorescence in tissue in the original state. (b) YFP fluorescence in tissue in the original state. (c) DsRed fluorescence in tissue in the original state. (d) GFP fluorescence following embedding with neutral resin. (e) YFP fluorescence following embedding with neutral resin. (f) DsRed fluorescence following embedding with neutral resin. (g) Pixel intensities of GFP fluorescence indicated by the red (d) and blue (a) lines are plotted as curves of the corresponding color. (h) Pixel intensities of YFP fluorescence indicated by the red (e) and blue (b) lines are plotted as curves of the corresponding color. (i) Pixel intensities of DsRed fluorescence indicated by the red (f) and blue (c) lines are plotted as curves of the corresponding color. (j) Preservation ratios of GFP, YFP, and DsRed. Scale bar (a)-(f): $30 \mu \mathrm{m}$. Confocal fluorescence microscopy (Zeiss 780), objective lens $20 \times \mathrm{W}$, NA 1.0 , zoom $1, z$-axis interval $1 \mu \mathrm{m}$. 

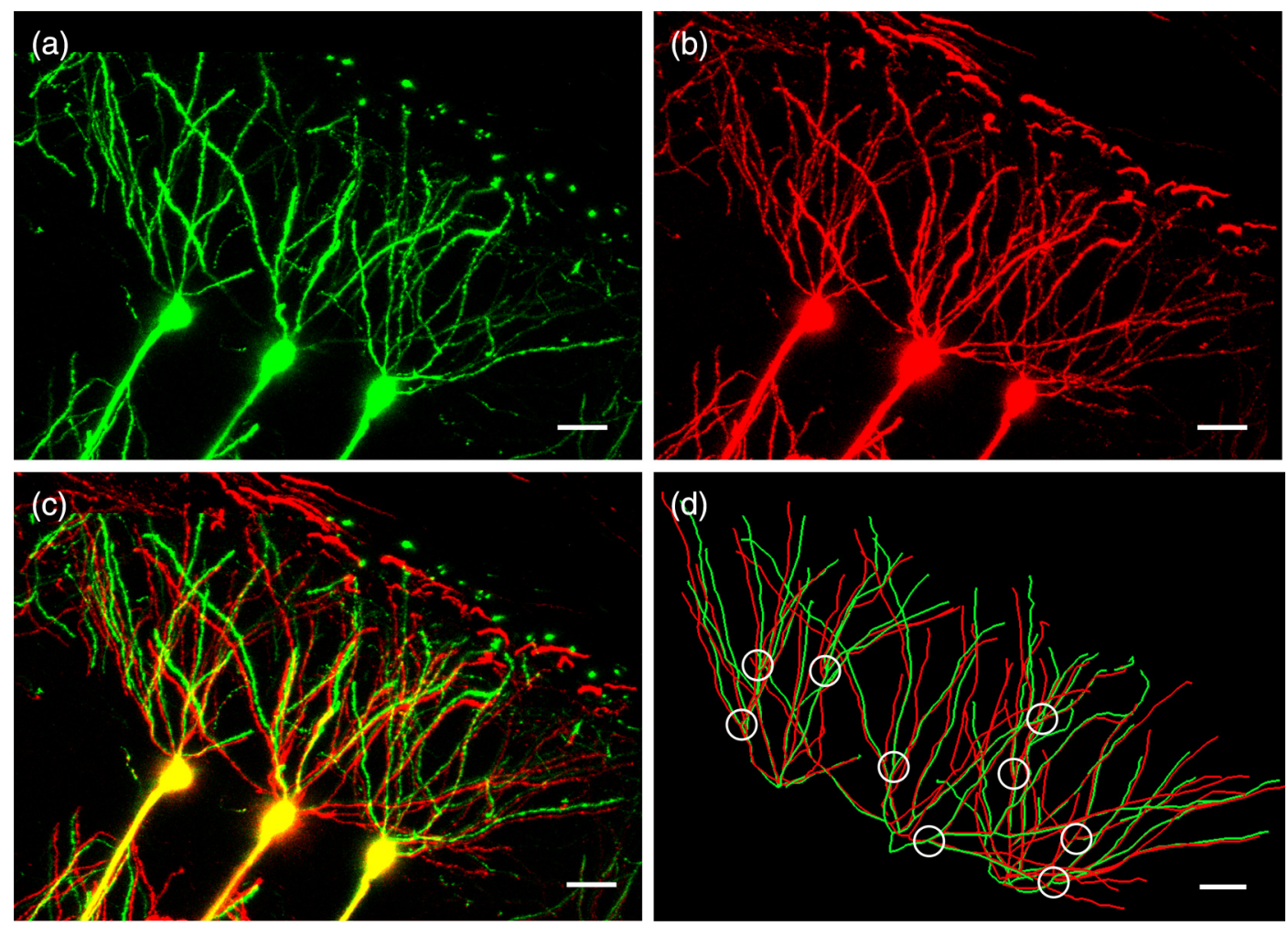

Fig. 3 Morphological analysis of nerve structure before and after neutral resin embedding using the data in Figs. 2(a) and (d). (a) GFP fluorescence in tissue in the original state. (b) GFP fluorescence following embedding with neutral embedding resin. The original image was enlarged 1.38 times and $x$-axis to the left by $173.9 \mu \mathrm{m}, y$-axis moves upward by $82.3 \mu \mathrm{m}$, rotate $3.13 \mathrm{deg}$ (rotate on the $x$-, $y$-plane) clockwise along the center of the image after embedding. (c) Merged image from (a) and (b). (d) The tracked image of morphological structure before and after neutral resin embedding from (a) and (b). Scale bar (a)-(d): $10 \mu \mathrm{m}$.

the Euclidean distance of each pair branch, then multiple measured branch distance of nerve structure and calculated average value, which is $1.91 \pm 1.1 \mu \mathrm{m}$ (Fig. 3). The distortion degree of the nerve fiber structure was characterized by the average
Euclidean distance, the result indicated that the polymerization rate is uniform and the nerve fibers did not appear to be obviously distorted, it means the change of morphological in biological tissue is slight.
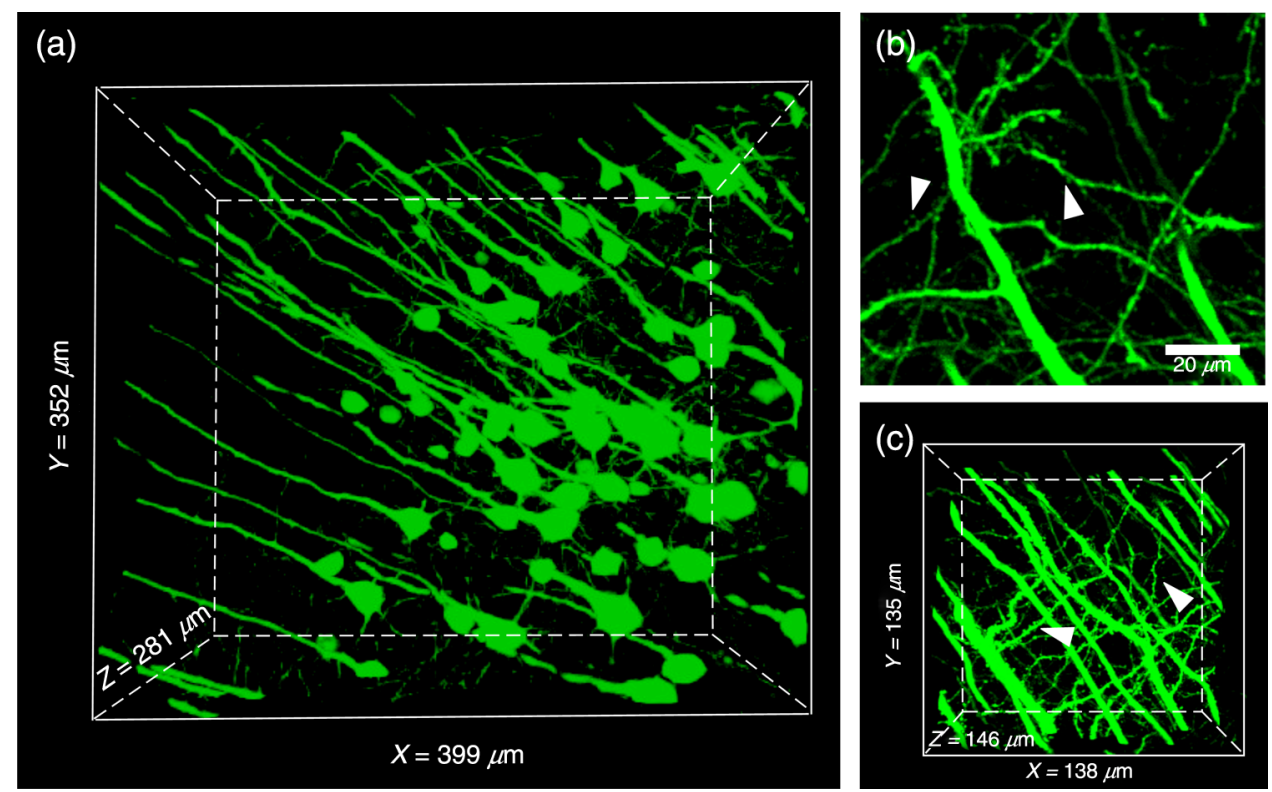

Fig. 4 Two-photon microscopy imaging of GFP-labeled whole brain. (a) Cortex nerve three-dimensional structure. (b) Magnified superposition of cortex nerve structure, scale bar $20 \mu \mathrm{m}$. (c) Magnified cortex nerve three-dimensional structure. Two-photon microscope (Nikon A1). Objective lens $25 \times \mathrm{W}$, NA 1.10 , zoom 2, laser $920 \mathrm{~nm}$, laser power $10 \%, z$-axis interval $1 \mu \mathrm{m}$. 
The whole brain was rinsed in PBS solution for $24 \mathrm{~h}$ and then gradient dehydrated at $4^{\circ} \mathrm{C}$ in the dark as follows: $50 \%$ THF for $2 \mathrm{~h}, 75 \%$ THF for $2 \mathrm{~h}, 100 \%$ THF for $2 \mathrm{~h}, 100 \%$ THF for $3 \mathrm{~h}$, and $100 \%$ THF for $3 \mathrm{~h}$. The dehydrated brain was then embedded in neutral embedding resin as follows: neutral embedding resin for $2 \mathrm{~h}$, neutral embedding resin for $24 \mathrm{~h}$, and neutral embedding resin for $48 \mathrm{~h}$ at $4^{\circ} \mathrm{C}$ in the dark. Next, the permeated mouse brain was placed in a capsule that was filled with neutral embedding resin. The curing procedure was as follows: $30^{\circ} \mathrm{C}$ for $8 \mathrm{~h}, 35^{\circ} \mathrm{C}$ for $8 \mathrm{~h}, 40^{\circ} \mathrm{C}$ for $8 \mathrm{~h}$, and $45^{\circ} \mathrm{C}$ for $8 \mathrm{~h}$ in vacuum and dark conditions. For optical imaging, the resin block of whole brain was sectioned via diamond knife and imaged the flat surface using two-photon microscopy. The results showed that neutral resin-embedded whole brain could be used for optical imaging (Fig. 4). The neuronal cell body and neurite protrusion structure could be seen clearly.

In this study, the random polymerization of glycidyl methacrylate and ethyl methacrylate was carried out using a free radical initiator to generate a rigid main chain structure. The reaction produced hydroxyl groups through the epoxy group of glycidyl methacrylate reacted with residual amine groups of biological tissue in the presence of the catalyst triphenylphosphine. This reaction improved the compatibility between the biological tissue and the embedding resin. Ethylene glycol dimethacrylate acted as the crosslinking agent to induce the formation of a crosslinked network structure, which was beneficial for improving the registration accuracy of 3-D imaging of large samples by reducing the peristalsis of polymer chains. The main factors mediating the preservation of fluorescent proteins are the
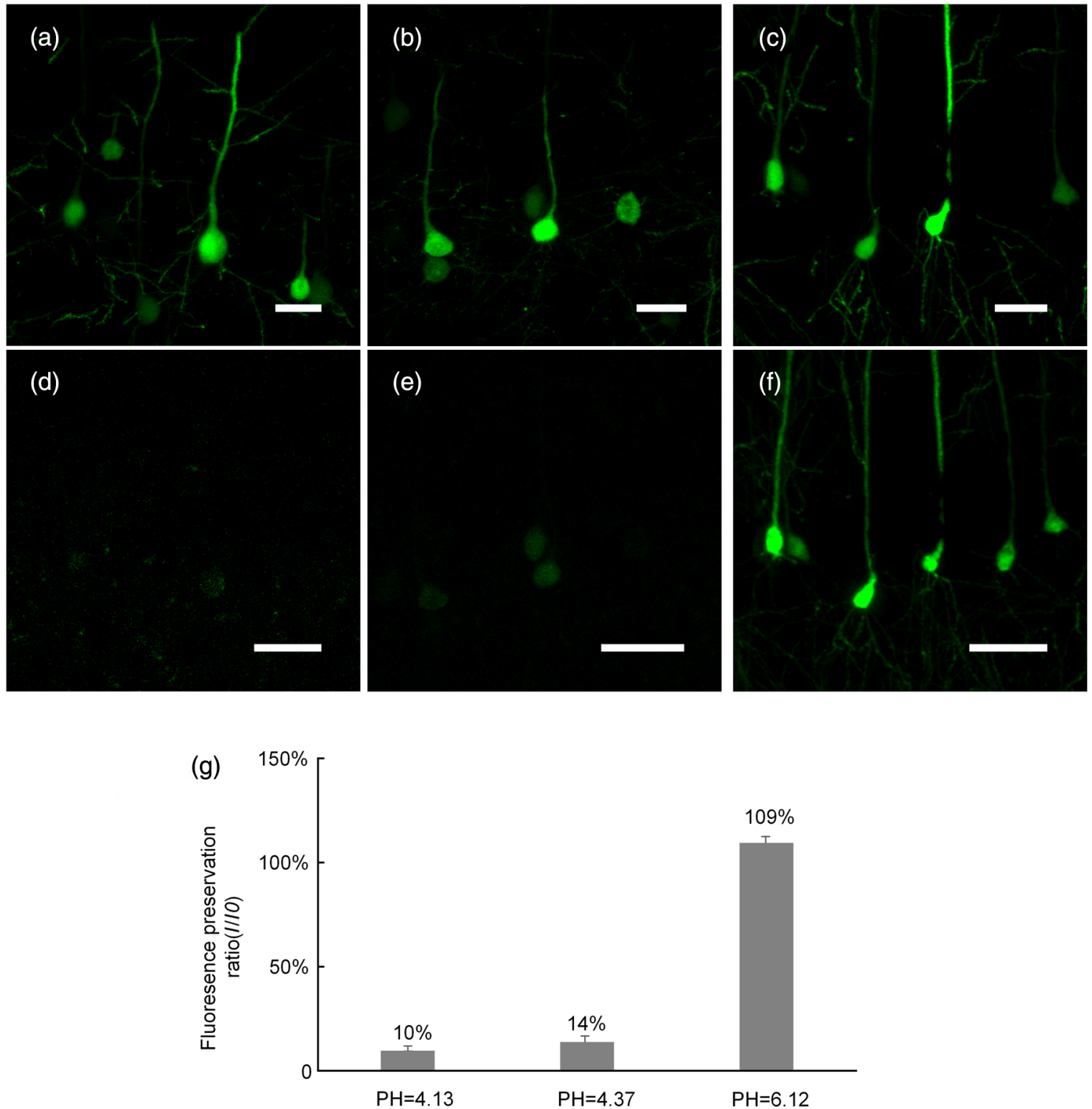

Fig. 5 Preservation ratio of GFP labeled brain slice embedded in this neutral resin with different $\mathrm{pH}$ value adjustment. (a)-(c) GFP fluorescence in tissue in the original state and in embedded states with $\mathrm{pH}$ value (d) 4.13, (e) 4.37, and (f) 6.12. (g) Preservation ratio of GFP after different $\mathrm{pH}$ value resin embedded. Scale bar (a)-(f): $30 \mu \mathrm{m}$. Confocal fluorescence microscopy (Zeiss 780 ), objective lens $20 \times \mathrm{W}, \mathrm{NA} 1.0$, zoom 1, $z$-axis interval $1 \mu \mathrm{m}$. 
conjugated $\pi$-electron cloud density of the fluorescent chromophore and its planar rigid structure. ${ }^{28,29}$ GFP and its variants combine with hydrogen ions in acidic environments to reduce the conjugated $\pi$-electron cloud density of fluorescent chromophore, and polar solvents and temperature also affect the planar rigid structure of GFP fluorescent chromophore, which could reversibly or irreversibly quench fluorescence. ${ }^{30-32}$

Commercial GMA (2-hydroxyethyl methacrylate: $67 \mathrm{~g}, \mathrm{n}$ butyl methacrylate: $30 \mathrm{~g}$, benzoyl peroxide paste: $0.6 \mathrm{~g}$ ) resin can maintain $70 \%$ of the fluorescence of GFP after embedding, representing the best result for a commercial resin embedding reagent. The fluorescence preservation ratio for GFP with the neutral resin was $126.5 \%$, whereas those for YFP and DsRed were $155.8 \%$ and $218.4 \%$, respectively. This result could be explained fundamental cause of fluorescence preservation by the $\mathrm{pH}$ value of the acrylic monomers, which was 6.38 , indicating that in this near-neutral environment, few hydrogen ions could combine with the fluorescent chromophore of GFP or its variants to reduce the conjugated $\pi$-electron cloud density to quench fluorescence. In order to prove this point, we adjusted the $\mathrm{pH}$ value of the neutral embedding resin through adding acetic acid and then embedded GFP brain slices, and examined the intensity of fluorescence. The method of quantitative analysis of fluorescence preservation ratio is the same as the above method. The shrinkage ratio is similar after the same resin embedding, the results showed that the lower the $\mathrm{pH}$ value, the more the GFP molecules in dark state (Fig. 5). The commercial resins LR white ( $\mathrm{pH} 4.80$ ) and Unicryl ( $\mathrm{pH}$ 5.12) quench endogenous fluorescence because the $\mathrm{pH}$ of these monomers is acidic. To solve the problem of endogenous fluorescence quenching after resin embedding, an organic base can be added to the commercial resin, and the polymerization inhibitor 4-methoxyphenol can be removed from the monomers to increase the $\mathrm{pH}$ value. However, this method leads to a low degree of polymerization and disrupts polymerization when the content of ethanolamine is more than $1 \%$, and the resin block becomes brittle and difficult to section. ${ }^{18,19}$ Alternatively, the fluorescence of the embedded sample can be reactivated in lye; however, this method reactivates the fluorescence of biological tissue on the surface of the embedding resin rapidly, whereas reactivation of the fluorescence inside the embedding resin takes several days. Depolymerization and swelling of the resin occur after several days of lye immersion, causing the resin to become soft. ${ }^{20}$

In contrast with the above methods, the endogenous fluorescence preservation ratio after embedding of the neutral resin was almost twice that of commercial resin GMA, and the resulting samples had adequate hardness (shore hardness: 78-80 D) for sectioning of large samples. The following main factor has increased the signal intensity and capacity: the shrinkage ratio of biological tissue is about $20 \%$ to $30 \%$ after dehydration, and this would cause obvious aggregation of the fluorescent molecules. ${ }^{33}$ The results from two-photon fluorescence microscopy imaging showed that the fluorescence signal and neuronal fiber structure were well preserved with the neutral resin, and the neuronal fibers were not distorted. Thus, the reaction rate was uniform, and this method could be applied for the embedding of large samples.

In summary, we developed a neutral embedding resin that could be used to preserve endogenous fluorescence and adequate hardness, without the requirement for adding an organic base to adjust the $\mathrm{pH}$ value of monomers before polymerization or reactivate fluorescence in lye after polymerization. Accordingly, our findings demonstrated that this resin may have applications in the embedding of large samples for fluorescence imaging.

\section{Disclosures}

The authors have no relevant financial interests in this article and no potential conflicts of interest to disclose.

\section{Acknowledgments}

We thank Qi Zhang, Fangfang Yin, Can Zhou, Ning Li, Pei Li, Ke Bai, and Gong Rao for assistance with the experiments and imaging. We thank Yuanlei Yue and other members of the Britton Chance Center for Biomedical Photonics for their assistance. This work was supported by the National Basic Research Program of China (Grant No. 2015CB755603) and NSFC projects (Grant No. 61421064).

\section{References}

1. P. Osten and T. W. Margrie, "Mapping brain circuitry with a light microscope," Nat. Methods 10, 515-523 (2013).

2. S. Mikula, J. Binding, and W. Denk, "Staining and embedding the whole mouse brain for electron microscopy," Nat. Methods 9, 11981201 (2012).

3. G. Newman and J. Hobot, "Resins for combined light and electron microscopy: a half century of development," Histochem. J. 31, 495505 (1999).

4. T. Ragan et al., "Serial two-photon tomography for automated ex vivo mouse brain imaging," Nat. Methods 9, 255-258 (2012).

5. G. Knott et al., "Serial section scanning electron microscopy of adult brain tissue using focused ion beam milling," J. Neurosci. 28, 29592964 (2008).

6. K. D. Micheva and S. J. Smith, "Array tomography: a new tool for imaging the molecular architecture and ultrastructure of neural circuits," Neuron 55, 25-36 (2007).

7. A. Li et al., "Micro-optical sectioning tomography to obtain a high-resolution atlas of the mouse brain," Science 330, 1404-1408 (2010).

8. A. R. Spurr, "A low-viscosity epoxy resin embedding medium for electron microscopy," J. Ultrastruct. Res. 26, 31-43 (1969).

9. B. L. Armbruster et al., "Specimen preparation for electron microscopy using low temperature embedding resins," J. Microsc. 126, 77-85 (1982).

10. K. L. McDonald, "Rapid embedding methods into epoxy and LR white resins for morphological and immunological analysis of cryofixed biological specimens," Microsc. Microanal. 20, 152-163 (2014).

11. J. J. Mozdzen and D. F. Keren, "Detection of immunoglobulin A by immunofluorescence in glycol methacrylate-embedded human colon," J. Histochem. Cytochem. 30, 532-535 (1982).

12. R. Yang et al., "Immunohistochemistry of matrix markers in Technovit 9100 new-embedded undecalcified bone sections," Eur. Cell Mater. 6, 57-71 (2003).

13. J. D. Acetarin, E. Carlemalm, and W. Villiger, "Developments of new Lowicryl® resins for embedding biological specimens at even lower temperatures," J. Microsc. 143, 81-88 (1986).

14. M. Steiner, C. Schöfer, and W. Mosgoeller, "In situ flat embedding of monolayers and cell relocation in the acrylic resin LR White for comparative light and electron microscopy studies," Histochem. J. 26, 934938 (1994).

15. D. R. Keene et al., "Confocal/TEM overlay microscopy: a simple method for correlating confocal and electron microscopy of cells expressing GFP/YFP fusion proteins," Microsc. Microanal. 14, 342348 (2008).

16. W. Kukulski et al., "Correlated fluorescence and 3D electron microscopy with high sensitivity and spatial precision," J. Cell Biol. 192, 111-119 (2011).

17. E. H. Leduc and S. J. Holt, "Hydroxypropyl methacrylate, a new watermiscible embedding medium for electron microscopy," J. Cell Biol. 26, 137-155 (1965). 
18. Z. Yang et al., "Development of a plastic embedding method for largevolume and luorescent-protein-expressing tissues," PLoS One 4, 1-5 (2013).

19. K. Bell et al., "Correlative imaging of fluorescent proteins in resinembedded plant material," Plant Physiol. 161, 1595-1603 (2013).

20. H. Xiong et al., "Chemical reactivation of quenched fluorescent protein molecules enables resin-embedded fluorescence microimaging," Nat. Commun. 5, 3992 (2014).

21. K. Chung et al., "Structural and molecular interrogation of intact biological systems," Nature 497, 332-337 (2013).

22. F. Chen, P. W. Tillberg, and E. S. Boyden, "Expansion microscopy," Science 347, 543-548 (2015).

23. E. Murray et al., "Simple, scalable proteomic imaging for high dimensional profiling of intact systems," Cell 163, 1500-1514 (2015).

24. J. Mijovic, A. Fishbain, and J. Wijaya, "Mechanistic modeling of epoxy-amine kinetics. 1. Model compound study," Macromolecules 25, 979-985 (1992).

25. N. Pal, A. Srivastava, and J. S. P. Rai, "Kinetics and mechanism of esterification of epoxy resin in presence of triphenylphosphine," Int. J. Chem. Kinet. 36, 280-285 (2004).

26. H. W. Dong, The Allen Reference Atlas: a Digital Color Brain Atlas of the C57Bl/6J Male Mouse, John Wiley \& Sons Inc., Chichester, West Sussex (2008).

27. Y. Gang et al., "Embedding and chemical reactivation of green fluorescent protein in the whole mouse brain for optical micro-imaging," Front. Neurosci. 11, 121 (2017).
28. R. Y. Tsien, "The green fluorescent protein," Аппи. Rev. Biochem. 67, 509-544 (1998).

29. N. C. Shaner, P. A. Steinbach, and R. Y. Tsien, "A guide to choosing fluorescent proteins," Nat. Methods 2, 905-909 (2005).

30. M. Kneen et al., "Green fluorescent protein as a noninvasive intracellular pH indicator," Biophys. J. 74, 1591-1599 (1998).

31. M. Zimmer, "Green fluorescent protein (GFP): applications, structure, and related photophysical behavior," Chem. Rev. 102, 759-782 (2002).

32. S. M. Levchenko, A. Pliss, and J. Qu, "Fluorescence lifetime imaging of fluorescent proteins as an effective quantitative tool for noninvasive study of intracellular processes," J. Innovative Opt. Health Sci. 11, 1730009 (2017)

33. C. Pan et al., "Shrinkage-mediated imaging of entire organs and organisms using uDISCO," Nat. Methods 13, 859-867 (2016).

Hongfu Zhou received his MSc degree in polymer chemistry and physics from Hubei Institute of Chemistry. He is studying for his $\mathrm{PhD}$ in biomedical engineering under the supervision of professor Shaoqun Zeng at Huazhong University of Science and Technology. His research interests are mainly focused on biopolymer materials and fluorescent molecule switch.

Biographies for the other authors are not available. 